people eligible. Across Wales the eligible population could vary from 493,000 using an age range of 50-74 years and a broad search strategy, down to 161,000 using an age range of 60-74 years and limiting the search strategy to current and more recent ex-smokers. As radiology reporting capacity may be the limiting factor for a national programme, focussing invitations to those at highest risk may be desirable in the early stages of project development.

\section{P163 PREPARING WALES FOR LUNG CANCER SCREENING - UPDATING GP RECORD SMOKING DATA USING AN AUTOMATED TEXT MESSAGE SYSTEM}

${ }^{1}$ JS Engela-Volker, ${ }^{2}$ SR Eccles. ${ }^{1}$ Cardiff University, Neuadd Meirionnydd, Cardiff, UK; ${ }^{2}$ Royal Glamorgan Hospital, Cwm Taf Morgannwg, Cardiff, UK

\subsection{6/thorax-2021-BTSabstracts.272}

Introduction Targeted lung cancer screening requires identification of current and ex-smokers. In the NHS England Targeted Lung Health Check Programme this relies on smoking history data in GP records. There are concerns about the completeness and accuracy of this data, meaning some eligible people may not be invited. We interrogated GP records at several practices in Wales to examine the completeness of smoking data and used an automated text message system to update smoking data for people with no smoking status recorded.

Methods GP records for patients aged 50 to 74 years $(+364$ days) were searched for the collective tobacco ' 137 ' Read code. Patients with no tobacco-related code were sent a standardised text message with the option of three replies to indicate their smoking status. On successful reply, a tobacco Read code was automatically recorded in the patient record, and a confirmation text including a link to 'Help Me Quit' was sent to the patient.

Results Across six GP practices, 670/20,402 patients (3.3\%) aged 50-74 had no tobacco code recorded. Of these, 293 (43.7\%) had a validated mobile phone number recorded, of whom 166/293 (56.7\%) successfully replied to the text. Of these, $71 / 166(42.8 \%)$ were current or ex-smokers, including $21(12.7 \%)$ current smokers.

Conclusions An automated text message system was used to successfully update smoking data in patients with no smoking status recorded. Of the respondents, almost half proved to be current or ex-smokers who would be eligible for a Targeted Lung Health Check. This system could be used to improve the completeness of GP records smoking data, particularly as a resource-sparing method at practices with lower levels of data completeness.

\section{P164 OUTCOME OF LUNG NODULE SURVEILLANCE: A BRIEF RETROSPECTIVE REVIEW OF A COHORT OF PATIENTS FOLLOWED-UP ACCORDING TO BTS GUIDELINES}

${ }^{1} \mathrm{~A}$ Perez Augusto, ${ }^{2} \mathrm{~S}$ Datta, ${ }^{2} \mathrm{Y}$ Ling, ${ }^{2} \mathrm{~L}$ McClure, ${ }^{2} \mathrm{D}$ Grieve, ${ }^{2} \mathrm{M}$ Majury. ${ }^{1}$ University of Glasgow, Glasgow, UK; ${ }^{2}$ Royal Alexandra Hospital, Paisley, UK

\subsection{6/thorax-2021-BTSabstracts.273}

Introduction Lung cancer continues to be the leading cause of cancer death in the UK and the 2nd most common cancer diagnosed each year. In 2015, the British Thoracic Society (BTS) published the updated guidelines on lung nodule

\section{STAGE AT DIAGNOSIS: GENERAL POPULATION VS BTS NODULE FOLLOW-UP}

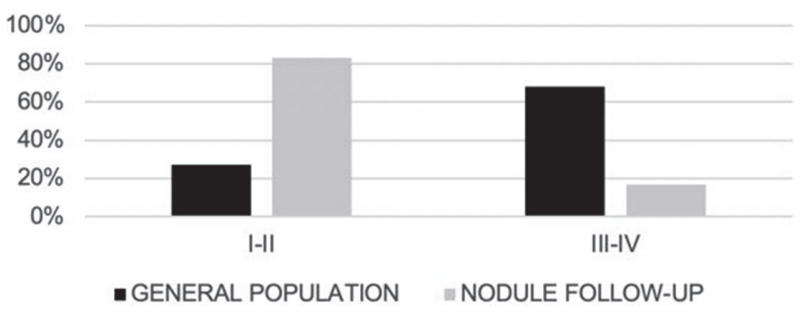

Abstract P164 Figure 1 Comparison between the stages at diagnosis in patients in surveillance programme(grey) vs those in the general population (black)

follow-up with the aim of improving early identification of lung malignancies and improving survival. Here we aim to determine the rate of early cancer detection in patients referred to the lung nodule MDT and explore their outcomes. Methods Patients were identified from the minutes of lung nodule MDTs celebrated during 2018 and information gathered via RIS, PACS and clinical record systems of the hospital.

Results 92 patients were identified using the method described above. The median age of participants was 67.5 (42-88) and there were slightly more females than males (51 to $41 ; 55.4$ to $44.6 \%)$. The rate of compliance with guidelines was very high at $98.9 \%$. We identified 6 lung primary malignancies of which all but 1 (83.3\%) were in early stages (stage I or II), compared to $27 \%$ in lung cancers detected in the population (figure 1). Of the patients with early-stage lung cancer, 3 were judged not fit for active treatment. The other 2 underwent lobectomies and have had more than 2 disease-free years as of the time of this writing.

Conclusion This data shows that implementation of the BTS guidelines in lung nodule follow-up leads to higher rates of early-stage lung cancer detection and improved prognosis for these patients. However, we also note that a relatively high proportion of these detected lung cancers are found in patients with poor functional status making them ineligible for active treatment. We propose that it may be beneficial for future editions of this BTS guidelines to explore including clear entry and retainment criteria for patients which, no matter the outcome, will be unable to withstand active treatment. These could include looking at, for example, elements such as minimum functional or pulmonary status. This would avoid some patients undergoing a 2 to 4 -year-long surveillance programme which will ultimately not affect their management options.

\section{P165 THE 'SUSPICIOUS' CHEST X-RAY. HOW GOOD ARE WE AT DISTINGUISHING HIGH RISK FROM LOW RISK ABNORMALITIES?}

JH Noble, A Rehman, H Steer. Gloucestershire Hospitals NHS Foundation Trust, Gloucester, UK

\subsection{6/thorax-2021-BTSabstracts.274}

Introduction We have developed two parallel direct recall CT pathways for patients with abnormal CXRs - a CX3 (NOLCP) pathway where CXR is suspicious for lung cancer, 